\title{
A cross-sectional study examining the associations between shift work and diet quality
}

\author{
N. Hornzee ${ }^{1}$, C. O'Connor ${ }^{2}$, N. Byrne ${ }^{2}$, M. Whelan ${ }^{3}$ and R. Gibson ${ }^{2}$ \\ ${ }^{1}$ Department of Life Sciences, University of Roehampton, London, UK, \\ ${ }^{2}$ Department of Nutritional Sciences, School of Life Course Sciences, King's College London, London, UK and \\ ${ }^{3}$ Work Ready Programme, British Dietetic Association, Birmingham, UK
}

Shift workers, particularly those working rotating and night shifts, are at an increased risk of diabetes ${ }^{(1)}$ and cardio- metabolic diseases ${ }^{(2)}$. Shift work impacts health behaviours like diet, which may contribute to poor health outcomes in this population. Limited studies have investigated diet quality and sources of food in shift workers. The aims of this cross- sectional study were to examine associations between types of shift work and i) reported diet quality and ii) use of vending machines and packed lunches, in a population of free-living individuals.

A convenience sample of 2063 UK employees completed a web-based anonymised survey that was distributed via Union of Shop, Distributive and Allied Workers, Kerry Foods Ltd., King's College London and the British Dietetic Association between March and April 2019 (King's Research Ethics Committee MRA-18/19-9228). Employees were categorised into 5 working groups: day, early, late, night and mixed shift. A diet quality score (DQS) was calculated using the 20 -item Leeds Short-Form FFQ ${ }^{\text {(3) }}$, with 12 points or more representing a healthy diet. Daily intake of sugar-sweetened beverages (SSBs) was also recorded (multiples of $250 \mathrm{ml}$ ). Using a 5-point Likert scale, participants reported vending machine and packed lunch use at work. Student's T-Test, ANOVA and Chi ${ }^{2}$ assessed the association between shift type and diet quality, and use of vending machines and packed lunches.

Day workers $(n=1014)$ reported a higher mean DQS $(10.09$, SD 1.96) compared to all categories of shift workers $(9.66$, SD $1.99, p$ $<.0001$ ), with the greatest difference compared to night workers $(9.37$, SD 2.04). The greatest difference in fruit ( $\mathrm{p}<0.0001)$ and vegetable $(\mathrm{p}=0.003)$ intake was shown between day and night shift workers. Day workers were more likely not to consume SSBs compared to night workers (71.6\% vs. 59.5\%) and night workers were more likely to consume $750 \mathrm{ml}$ or more of SSBs per day compared to day workers $(15 \%$ vs. $5.5 \%, \mathrm{p}<0.0001)$. Vending machine use was significantly associated with shift type $(\mathrm{p}<0.0001)$, with $11.8 \%$ of night workers always using vending machines, compared with $3.8 \%$ of day workers. Night shift workers were most likely (29.4\%) to always bring a packed lunch to work, compared to $21.9 \%$ of day workers.

Shift work, especially night shift work, was associated with a lower DQS and higher intakes of SSBs. Similarly, night shift workers were most likely to always use vending machines, potentially contributing to poorer diet quality. Further research is needed to investigate the health profile of packed lunch usage in night workers and understand how diet behaviours differ between work environments in shift workers.

\section{References}

1. Gan Y, Yang C, Tong X et al. (2015) Occup Environ Med 2, 72-78.

2. Wang $\mathrm{F}$, Zhang L, Zhang Y et al. (2014) Obes Rev 15, 709-720.

3. Cleghorn CL, Harrison R, Ransley J et al. (2016) Public Health Nutr 19, 2915-2923. 